\title{
HYPERSTABILITY OF RASSIAS-RAVI RECIPROCAL FUNCTIONAL EQUATION
}

\author{
B. V. SENTHIL KUMAR, KHALIFA AL-SHAQSI, AND HEMEN DUTTA
}

Received 01 January, 2020

\begin{abstract}
The investigation of stabilities of various types of equations is an interesting and evolving research area in the field of mathematical analysis. Recently, there are many research papers published on this topic, especially mixed type and multiplicative inverse functional equations. We propose a new functional equation in this study which is quite different from the functional equations already dealt in the literature. The main feature of the equation dealt in this study is that it has two different solutions, namely additive and multiplicative inverse functions. We also prove that the hyperstability results hold good for this equation.
\end{abstract}

2010 Mathematics Subject Classification: 39B82; 39B72

Keywords: additive function, multiplicative inverse function, Ulam-Hyers stability, hyperstability

\section{INTRODUCTION}

The analysis of stability of Functional Equation (FE) is due to the celebrated query presented in [32]. An excellent response was presented in [15]. This method of proving stability result of FE is termed as Hyers-Ulam (H-U) stability which involves a small positive constant as upper bound. Later, this result influenced many researchers to solve stability problems via different directions in $[14,20,21]$ and these results are respectively called as generalized Hyers-Ulam-Rassias stability, Ulam-GavrutaRassias (U-G-R) stability and Hyers-Ulam-Rassias (H-U-R) stability.

For the first time, the hyperstability results associated with the ring homomorphisms were obtained in [5]. Also, the hyperstability of a class of linear functional equations were dealt in [18]. There are a number of published papers associated with hyperstability results and stability results via fixed point technique of various FEs, one may refer to $[1-3,6-10,13,17,19,25]$.

The first two authors are supported by The Research Council, Oman (Under Project proposal ID: $\mathrm{BFP} / \mathrm{RGP} / \mathrm{CBS} / 18 / 099)$. 
On the other hand, for the first time in this theory, a rational FE of the form

$$
r(x+y)=\frac{r(x) r(y)}{r(x)+r(y)}
$$

was introduced and studied its stability results in [23], where $r: \mathbb{R}^{\star} \longrightarrow \mathbb{R}$ is a mapping. It is interesting to note that the rational function $f(x)=\frac{c}{x}, c$ being a constant, is a solution of equation (1.1). Motivated by the equation (1.1), there are numerous papers published on the problems of solving stability of various multiplicative inverse FEs of the type reciprocal-quadratic, reciprocal-cubic, reciprocal-quintic, etc., and radical functional equation. The detailed information regarding these results are available in $[4,11,12,16,22,24,26-31]$.

In an algebraic polynomial equation $g(x)=0$, if we replace the variable $x$ by $\frac{1}{x}$ and if we get the same equation, then it is called a reciprocal equation. Also, if $\alpha$ is a root of $g(x)=0$, then $\frac{1}{\alpha}$ is also a root of $g(x)=0$.

These concepts together with the results of equation (1.2) instigated us to deal with a new FE of the form

$$
h\left(\sum_{j=1}^{m} u_{j}\right)+h\left(\frac{\prod_{j=1}^{m} u_{j}}{\sum_{j=1}^{m} \prod_{k=1, k \neq j}^{m} u_{k}}\right)=\frac{\prod_{j=1}^{m} h\left(u_{j}\right)}{\sum_{j=1}^{m} \prod_{k=1, k \neq j}^{m} h\left(u_{k}\right)}+\sum_{j=1}^{m} h\left(u_{j}\right) .
$$

One can easily verify that the functions $h(u)=u$ and $h(u)=\frac{1}{u}$ are solutions of equation (1.2). We present preliminaries and some basic results connected with (1.2). We also establish hyperstability results of (1.2) in the setting of real numbers.

Thoughout this paper, let $\mathbb{N}, \mathbb{N}_{0}, \mathbb{N}_{m_{0}}, \mathbb{R}$ and $\mathbb{R}^{\star}$ denote the set of all natural numbers, the set of all nonnegative integers, the set of all integers greater than or equal to $m_{0}$, the set of all real numbers and the set of all non-zero real numbers, respectively.

\section{Preliminaries}

Here we recall some significant concepts related with hyperstability and fixed point theorem [8] which are useful to prove our main results of this investigation. The ensuing three propositions are significant in obtaining the hyperstability results.

(P1) Let $A$ and $B$ be a nonempty set and a Banach space, respectively.

Let $h_{1}, h_{2}, \ldots, h_{k}: A \longrightarrow A$ and $Q_{1}, Q_{2}, \ldots, Q_{k}: A \longrightarrow \mathbb{R}_{+}$be given mappings.

(P2) Let an operator $\eta: B^{A} \longrightarrow B^{A}$ satisfies the inequality

$$
\|\eta \alpha(u)-\eta \beta(u)\| \leq \sum_{i=1}^{k} Q_{i}(u)\left\|\alpha\left(h_{i}(u)\right)-\beta\left(h_{i}(u)\right)\right\|
$$

for all $\alpha, \beta \in B^{A}, u \in A$. 
(P3) The mapping $\Gamma: \mathbb{R}_{+}^{A} \longrightarrow \mathbb{R}_{+}^{A}$ be defined by

$$
\Gamma \Delta(u)=\sum_{i=1}^{k} Q_{i}(u) \Delta\left(h_{i}(u)\right), \quad \Delta \in \mathbb{R}_{+}^{A}, u \in A .
$$

The subsequent theorem is employed in our investigation to claim the persistence of the distinct fixed point operator $\eta: B^{A} \longrightarrow B^{A}$.

Theorem 1. Let the propositions $(\mathrm{P} 1)-(\mathrm{P} 3)$ be substantial. Suppose the mappings $\psi: A \longrightarrow \mathbb{R}_{+}$and let $\phi: A \longrightarrow B$ satisfy the ensuing two conditions:

$$
\begin{gathered}
\|\eta \phi(u)-\phi(u)\| \leq \psi(u), \quad u \in A, \\
\psi^{\star}(u)=\sum_{n=0}^{\infty} \Gamma^{n} \psi(u)<\infty, \quad u \in A .
\end{gathered}
$$

Then, there exists a unique fixed point $\chi$ of $\eta$ such that

$$
\|\psi(u)-\chi(u)\| \leq \psi^{\star}(u), \quad u \in A .
$$

Furthermore,

$$
\chi(u)=\lim _{n \rightarrow \infty} \eta^{n} \psi(u)
$$

\section{BASIC SignifiCANT RESUlts CONNECTED With EQUATION (1.2)}

The following definition will be useful to prove our main results.

Definition 1. A function $h: \mathbb{R}^{\star} \longrightarrow \mathbb{R}$ is said to be a Rassias-Ravi reciprocal function if it satisfies the following general FE:

$$
h(m u)+h\left(\frac{u}{m}\right)=\frac{m^{2}+1}{m} h(u)
$$

for all $u \in \mathbb{R}^{\star}$ and any integer $m$.

Remark 1. From the above definition, it is clear that (1.2) satisfies (3.1) by plugging $u_{k}=u$, for $k=1,2, \ldots, m$ in (1.2). Hence (1.2) is said to be Rassias-Ravi reciprocal functional equation.

Remark 2. When $m=2$, (1.2) produces the following equation in two variables:

$$
h\left(u_{1}+u_{2}\right)+h\left(\frac{u_{1} u_{2}}{u_{1}+u_{2}}\right)=\frac{h\left(u_{1}\right) h\left(u_{2}\right)}{h\left(u_{1}\right)+h\left(u_{2}\right)}+\left[h\left(u_{1}\right)+h\left(u_{2}\right)\right] .
$$

When $m=3$, (1.2) induces the ensuing equation in three variables:

$$
\begin{aligned}
h\left(u_{1}+u_{2}+\right. & \left.u_{3}\right)+h\left(\frac{u_{1} u_{2} u_{3}}{u_{1} u_{2}+u_{1} u_{3}+u_{2} u_{3}}\right) \\
& =\frac{h\left(u_{1}\right) h\left(u_{2}\right) h\left(u_{3}\right)}{h\left(u_{1}\right) h\left(u_{2}\right)+h\left(u_{1}\right) h\left(u_{3}\right)+h\left(u_{2}\right) h\left(u_{3}\right)}+\left[h\left(u_{1}\right)+h\left(u_{2}\right)+h\left(u_{3}\right)\right] .
\end{aligned}
$$


Remark 3. In this investigation, we assume that

$$
\sum_{j=1}^{m} u_{j}, \sum_{j=1}^{m} \prod_{k=1, k \neq j}^{m} u_{k}, \sum_{j=1}^{m} \prod_{k=1, k \neq j}^{m} h\left(u_{k}\right) \neq 0,
$$

for all $u_{j} \in \mathbb{R}^{\star}, j=1,2, \ldots, m$.

Theorem 2. A mapping $h: \mathbb{R}^{\star} \longrightarrow \mathbb{R}$ satisfying (1.2) also satisfies

$$
h\left(m^{p} u\right)+h\left(\frac{u}{m^{p}}\right)=\frac{m^{2 p}+1}{m^{p}} h(u)
$$

for all $u \in \mathbb{R}^{\star}$ and $p>0$ is an integer.

Proof. Firstly, let us consider $u$ for every $u_{j}, j=1,2, \ldots, m$ in (1.2) to obtain

$$
h(m u)+h\left(\frac{u}{m}\right)=\frac{m^{2}+1}{m} h(u)
$$

for all $u \in \mathbb{R}^{\star}$. Next, reinstating $u$ by $m u$ in 3.3 and then multiplying by $\frac{m^{2}+1}{m}$ on its both sides, we get

$$
\frac{m^{2}+1}{m} h\left(m^{2} u\right)+\frac{m^{2}+1}{m} h(u)=\frac{\left(m^{2}+1\right)^{2}}{m^{2}} h(m u)
$$

for all $u \in \mathbb{R}^{\star}$. On the other hand, replacing $u$ by $\frac{u}{m}$ in (3.3) and then multiplying by $\frac{m^{2}+1}{m}$ on its both sides, we obtain

$$
\frac{m^{2}+1}{m} h(u)+\frac{m^{2}+1}{m} h\left(\frac{u}{m^{2}}\right)=\frac{\left(m^{2}+1\right)^{2}}{m^{2}} h\left(\frac{u}{m}\right)
$$

for all $u \in \mathbb{R}^{\star}$. Now, adding (3.4) with (3.5) and simplifying further, we arrive at

$$
h\left(m^{2} u\right)+h\left(\frac{u}{m^{2}}\right)=\frac{m^{4}+1}{m^{2}} h(u)
$$

for all $u \in \mathbb{R}^{\star}$. Again, plugging $u$ by $m u$ in (3.6), we get

$$
h\left(m^{3} u\right)+h\left(\frac{u}{m}\right)=\frac{m^{4}+1}{m^{2}} h(m u)
$$

for all $u \in \mathbb{R}^{\star}$. Also, substituting $u$ by $\frac{u}{m}$ in (3.6), we obtain

$$
h(m u)+h\left(\frac{u}{m^{3}}\right)-\frac{m^{4}+1}{m^{2}} h\left(\frac{u}{m}\right)
$$

for all $u \in \mathbb{R}^{\star}$. Now, summing (3.7) and (3.8) and simplifying further to arrive at

$$
h\left(m^{3} u\right)+h\left(\frac{u}{m^{3}}\right)=\frac{m^{6}+1}{m^{3}} h(u)
$$

for all $u \in \mathbb{R}^{\star}$. Proceeding with similar arguments and employing mathematical induction, one can find for any $p>0$ integer,

$$
h\left(m^{p} u\right)+h\left(\frac{u}{m^{p}}\right)=\frac{m^{2 p}+1}{m^{p}} h(u)
$$


for all $u \in \mathbb{R}^{\star}$. This completes the proof.

\section{Hyperstability of equation (1.2)}

In this section, by employing the notions and fixed point theorem proposed in [8], we establish the hyperstability of (1.2). For the sake of of convenience, let us define the difference operator $D h\left(u_{1}, \ldots, u_{m}\right): \underbrace{\mathbb{R}^{*} \times \cdots \times \mathbb{R}^{*}}_{(m \text { times })} \longrightarrow \mathbb{R}$ as follows:

$$
\begin{aligned}
& \operatorname{Dh}\left(u_{1}, u_{2}, \ldots, u_{m}\right) \\
& \quad=h\left(\sum_{j=1}^{m} u_{j}\right)+h\left(\frac{\prod_{j=1}^{m} u_{j}}{\sum_{j=1}^{m} \prod_{k=1, k \neq j}^{m} u_{k}}\right)-\frac{\prod_{j=1}^{m} h\left(u_{j}\right)}{\sum_{j=1}^{m} \prod_{k=1, k \neq j}^{m} h\left(u_{k}\right)}-\sum_{j=1}^{m} h\left(u_{j}\right)
\end{aligned}
$$

for all $u_{1}, \ldots, u_{m} \in \mathbb{R}^{\star}$

Theorem 3. Let $k>0$ and $p<0$ be fixed constants. Let there exists $n_{0} \in \mathbb{N}$ with $n u \in \mathbb{R}^{\star}$ for $u \in \mathbb{R}^{\star}, n \in \mathbb{N}_{n_{0}}$. Suppose a mapping $h: \mathbb{R}^{\star} \longrightarrow \mathbb{R}$ satisfies the inequality

$$
\left|D h\left(u_{1}, u_{2} \ldots, u_{m}\right)\right| \leq k \sum_{j=1}^{m}\left|u_{j}\right|^{p}
$$

for all $u_{1}, \ldots, u_{m} \in \mathbb{R}^{\star}$. Then there exists a unique Rassias-Ravi reciprocal function $H: \mathbb{R}^{\star} \longrightarrow \mathbb{R}$ satisfying (1.2) and

$$
|H(u)-h(u)| \leq \frac{m^{2} k}{1-m^{1+p}-m^{1-p}+m^{2}}|u|^{p}
$$

for all $u \in \mathbb{R}^{*}$.

Proof. Firstly, let us plug $u_{j}=u$, for $j=1,2, \ldots, m$ in (4.1) and then multiply by $\frac{m}{m^{2}+1}$ on its both sides to get

$$
\left|\frac{m}{m^{2}+1} h(m u)+\frac{m}{m^{2}+1} h\left(\frac{u}{m}\right)-h(u)\right| \leq \frac{m^{2} k}{m^{2}+1}|u|^{p}
$$

for all $u \in \mathbb{R}^{\star}$. We can find that there exists an $m_{0} \in \mathbb{N}_{m_{0}}$ such that

$$
\frac{m^{p}}{\left(m^{2}+1\right)^{p}}<1 \text { for } m \geq m_{0} \text {. }
$$

Let $m \in \mathbb{N}_{m_{0}}$ be fixed integer. Let us denote

$$
\begin{gathered}
\eta \alpha(u)=\frac{m}{m^{2}+1} \alpha(m u)+\frac{m}{m^{2}+1} \alpha\left(\frac{u}{m}\right), \quad u \in \mathbb{R}, \quad \alpha \in \mathbb{R}^{\mathbb{R}^{\star}}, \\
\psi(u)=\frac{m^{2} k}{m^{2}+1}|u|^{p}, \quad u \in \mathbb{R}^{\star} .
\end{gathered}
$$

Using (4.5) and (4.6), inequality (4.3) can be written as

$$
|\eta h(u)-h(u)| \leq \psi(u), \quad u \in \mathbb{R}^{\star} .
$$


The operator is defined by the following:

$$
\Gamma \rho(u)=\frac{m}{m^{2}+1} \rho(m u)+\frac{m}{m^{2}+1} \rho\left(\frac{u}{m}\right), \quad \rho \in \mathbb{R}_{+}^{\mathbb{R}^{\star}}, u \in \mathbb{R}^{\star}
$$

has the form which is defined in (P3) with $k=2$ and $h_{1}(u)=m u, h_{2}(u)=\frac{u}{m}$ and $Q_{1}(u)=Q_{2}(u)=\frac{m}{m^{2}+1}$ for $u \in \mathbb{R}^{\star}$. Also, for each $\alpha, \beta \in \mathbb{R}^{\mathbb{R}^{\star}}, u \in \mathbb{R}^{\star}$,

$$
\begin{aligned}
|\eta \alpha(u)-\eta \beta(u)| & =\left|\frac{m}{m^{2}+1} \alpha(m u)+\frac{m}{m^{2}+1} \alpha\left(\frac{u}{m}\right)-\frac{m}{m^{2}+1} \beta(m u)-\frac{m}{m^{2}+1} \beta\left(\frac{u}{m}\right)\right| \\
& \leq \frac{m}{m^{2}+1}|(\alpha-\beta)(m u)|+\frac{m}{m^{2}+1}\left|(\alpha-\beta)\left(\frac{u}{m}\right)\right| \\
& \leq \sum_{i=1}^{2} Q_{i}(u)\left|(\alpha-\beta) h_{i}(u)\right|
\end{aligned}
$$

Since $\frac{m}{m^{2}+1}\left(\frac{m^{2 p}+1}{m^{p}}\right)<1$, we have

$$
\begin{aligned}
\psi^{\star}(u)=\sum_{n=0}^{\infty} \Gamma^{n} \psi(u) & =\sum_{n=0}^{\infty} \frac{m^{2} k}{m^{2}+1}\left(\frac{m}{m^{2}+1}\left(\frac{m^{2 p}+1}{m^{p}}\right)\right)^{n}|u|^{p} \\
& =\frac{m^{2} k}{1-m^{1+p}-m^{1-p}+m^{2}}|u|^{p} .
\end{aligned}
$$

Owing to Theorem 1 , there exists a unique solution $H: \mathbb{R}^{\star} \longrightarrow \mathbb{R}$ of the equation

$$
H(u)=\frac{m}{m^{2}+1} h(m u)+\frac{m}{m^{2}+1} h\left(\frac{u}{m}\right)
$$

such that the inequality (4.2) holds. Moreover,

$$
H(u)=\lim _{n \rightarrow \infty} \eta^{n} h(u) .
$$

In order to show that $H$ satisfies (1.2), we find that

$$
\left|\eta^{n} D h\left(u_{1}, u_{2}, \ldots, u_{m}\right)\right| \leq k\left(\frac{m}{m^{2}+1}\right)^{n}\left(\frac{m^{2 p}+1}{m^{p}}\right)^{n} \sum_{j=1}^{m}|u|^{p}
$$

for all $u_{1}, \ldots, u_{m} \in \mathbb{R}^{\star}$, and $n \in \mathbb{N}_{0}$. Suppose $n=0$, then (4.13) becomes (4.1). So, let us fix $n \in \mathbb{N}_{0}$ and suppose that (4.13) holds for $n$ and $u_{1}, \ldots, u_{m} \in \mathbb{R}^{\star}$. Then

$$
\begin{aligned}
& \left|\eta^{n+1} \operatorname{Dh}\left(u_{1}, u_{2}, \ldots, u_{m}\right)\right| \\
& =\mid \frac{m}{m^{2}+1} \eta^{n} h\left(\sum_{j=1}^{m} m u_{j}\right)+\frac{m}{m^{2}+1} \eta^{n} h\left(\sum_{j=1}^{m} \frac{u_{j}}{m}\right) \\
& \quad+\frac{m}{m^{2}+1} \eta^{n} h\left(\frac{\prod_{j=1}^{m} m u_{j}}{\sum_{j=1}^{m} \prod_{k=1, k \neq j}^{m} m u_{k}}\right)+\frac{m}{m^{2}+1} \eta^{n} h\left(\frac{\prod_{j=1}^{m} \frac{u_{j}}{m}}{\sum_{j=1}^{m} \prod_{k=1, k \neq j}^{m} \frac{u_{k}}{m}}\right)
\end{aligned}
$$




$$
\begin{aligned}
& -\frac{m}{m^{2}+1} \eta^{n}\left(\frac{\prod_{j=1}^{m} h\left(m u_{j}\right)}{\sum_{j=1}^{m} \prod_{k=1, k \neq j}^{m} h\left(m u_{k}\right)}\right)-\frac{m}{m^{2}+1} \eta^{n}\left(\frac{\prod_{j=1}^{m} h\left(\frac{u_{j}}{m}\right)}{\sum_{j=1}^{m} \prod_{k=1, k \neq j}^{m} h\left(\frac{u_{k}}{m}\right)}\right) \\
& -\frac{m}{m^{2}+1} \eta^{n} \sum_{j=1}^{m} h\left(m u_{j}\right)-\frac{m}{m^{2}+1} \eta^{n} \sum_{j=1}^{m} h\left(\frac{u_{j}}{m}\right) \mid \\
\leq & k\left(\frac{m}{m^{2}+1}\right)^{n}\left(\frac{m^{2 p}+1}{m^{p}}\right)^{n}\left[\frac{m}{m^{2}+1} \sum_{j=1}^{m}\left|u_{j}\right|^{p}+\frac{m}{m^{2}+1} \sum_{j=1}^{m}\left|\frac{u_{j}}{m}\right|^{p}\right] \\
\leq & k\left(\frac{m}{m^{2}+1}\right)^{n+1}\left(\frac{m^{2 p}+1}{m^{p}}\right)^{n+1} \sum_{j=1}^{m}\left|u_{j}\right|^{p} .
\end{aligned}
$$

Hence through induction method, the above inequality (4.14) implies that (4.13) holds good for all $u_{j} \in \mathbb{R}^{\star}$, for $j=1,2, \ldots, m$. By letting $\mathrm{n}$ to $\infty$ in (4.13), we can find that $H$ satisfies (1.2). This completes the proof.

In the sequel, we provide two examples for the non-stability of equation (1.2).

Example 1. Let $A=[-1,1] \backslash\{0\}$ and let $h: A \longrightarrow \mathbb{R}$ be defined by $h(u)=u, u \in A$. Then for $u_{j} \in A, j=1,2, \ldots, m$ such that

$$
\begin{gathered}
\sum_{j=1}^{m} u_{j}, \sum_{j=1}^{m} \frac{u_{j}}{m}, \prod_{j=1}^{m} m u_{j}, \prod_{j=1}^{m} \frac{u_{j}}{m}, \frac{\prod_{j=1}^{m} m u_{j}}{\sum_{j=1}^{m} \prod_{k=1, k \neq j}^{m} m u_{k}}, \frac{\prod_{j=1}^{m} \frac{u_{j}}{m}}{\sum_{j=1}^{m} \prod_{k=1, k \neq j}^{m} \frac{u_{k}}{m}} \in A, \\
\left|D h\left(u_{1}, u_{2}, \ldots, u_{m}\right)\right| \leq \sum_{j=1}^{m}\left|u_{j}\right|^{p},
\end{gathered}
$$

with $p<0$, but $h$ does not satisfy (1.2).

The following theorem contains the hyperstability involving product of different powers of norms. The proof is obtained by similar arguments as in Theorem 3 . Hence we omit the proof and provide only the statement.

Theorem 4. Let $k>0$ be a fixed constant. Let $p_{j} \in \mathbb{R}, j=1,2, \ldots, m$ such that $p=\sum_{j=1}^{m} p_{i}<0$. Let $h: \mathbb{R}^{\star} \longrightarrow \mathbb{R}$ satisfy the following inequality

$$
\left|D h\left(u_{1}, u_{2}, \ldots, u_{m}\right)\right| \leq k \prod_{j=1}^{m}\left|u_{j}\right|^{p_{j}}
$$

for all $u_{j} \in \mathbb{R}^{\star}, j=1,2, \ldots, m$. Then there exists a unique Rassias-Ravi reciprocal function $H: \mathbb{R}^{\star} \longrightarrow \mathbb{R}$ satisfying (1.2) and

$$
|H(u)-h(u)| \leq \frac{m k}{1-m^{1+p}-m^{1-p}+m^{2}}|u|^{p}
$$

for all $u \in \mathbb{R}^{\star}$. 


\section{CONCLUSiON}

So far various forms of additive FEs and multiplicative inverse FEs are considered in this research field to obtain their stability results through different methods. For the first time, a new FE with additive function and multiplicative inverse function is proposed in this paper and its hyperstability results are proved via fixed point method.

\section{REFERENCES}

[1] L. Aiemsomboon and W. Sintunavarat, "On new hyperstability results for the generalized $p$-radical functional equation in quasi-Banach spaces with the illustrative example." Results in Fixed Point Theory and Applications, vol. 2019, no. 2019012, pp. 1-14, 2019.

[2] Y. Aribou, H. Dimou, and S. Kabbaj, "Generalized hyperstability of the cubic functional equation in ultrametric spaces." Linear and Topological Algebra, vol. 8, no. 2, pp. 97-104, 2019.

[3] A. Bahyrycz and M. Piszczek, "Hyperstability of the Jensen functional equation." Acta Math. Hungarica, vol. 142, no. 2, pp. 353-365, 2014, doi: 10.1007/s10474-013-0347-3.

[4] A. Bodaghi and B. V. Senthil Kumar, "Estimation of inexact reciprocal-quintic and reciprocalsextic functional equations." Mathematica, vol. 49, no. 82, pp. 3-14, 2017.

[5] D. G. Bourgin, "Approximately isometric and multiplicative transformations on continuous function rings." Duke Math. J., vol. 16, pp. 385-397, 1949.

[6] J. Brzdek, "Hyperstability of the Cauchy equation on restricted domains." Acta Math. Hungarica, vol. 141, no. 1-2, pp. 58-67, 2013, doi: 10.1007/s10474-013-0302-3.

[7] J. Brzdek, "Remarks on hyperstability of the Cauchy functional equations." Aequ. Math., vol. 86, pp. 255-267, 2013, doi: 10.1007/s00010-012-0168-4.

[8] J. Brzdek, J. Chudziak, and Z. Pales, "A fixed point approach to stability of functional equations." Nonlinear Anal., vol. 74, no. 17, pp. 6728-6732, 2011, doi: 10.1016/j.na.2011.06.052.

[9] J. Brzdek and K. Cieplinski, "A fixed point approach to the stability of functional equations in non-Archimedean metric spaces.” Nonlinear Anal., vol. 74, no. 18, pp. 6861-6867, 2011, doi: 10.1016/j.na.2011.06.050.

[10] L. Cadariu, L. Gavruta, and P. Gavruta, "Fixed points and generalized Hyers-Ulam stability." Abst. Appl. Anal., vol. 2012, no. 712743, pp. 1-12, 2012, doi: 10.1155/2012/712743.

[11] H. Dutta and B. V. Senthil Kumar, "Geometrical elucidations and approximation of some functional equations in numerous variables." Proc. Indian Natn. Sc. Acad., vol. 85, no. 3, pp. 603-611, 2019, doi: 10.16943/ptinsa/2019/49584.

[12] A. Ebadian, S. Zolfaghari, S. Ostadbashi, and C. Park, "Approximation on the reciprocal functional equation in several variables in matrix non-Archimedean random normed spaces." Adv. Difference. Equ., vol. 314, pp. 1-13, 2015, doi: 10.1186/s13662-015-0656-7.

[13] E. Gselmann, "Hyperstability of a functional equation." Acta Math. Hungarica, vol. 124, no. 1-2, pp. 179-188, 2009, doi: 10.1007/s10474-009-8174-2.

[14] P. Găvruta, "A generalization of the Hyers-Ulam-Rassias stability of approximately additive mapppings." J. Math. Anal. Appl., vol. 184, pp. 431-436, 1994, doi: 10.1006/jmaa.1994.1211.

[15] D. H. Hyers, "On the stability of the linear functional equation." Proc. Nat. Acad. Sci. U.S.A., vol. 27, pp. 222-224, 1941, doi: 10.1073/pnas.27.4.222.

[16] S. O. Kim, B. V. Senthil Kumar, and A. Bodaghi, "Stability and non-stability of the reciprocalcubic and reciprocal-quartic functional equations in non-Archimedean fields." Adv. Difference Equ., vol. 2017, no. 77, pp. 1-12, 2017, doi: 10.1186/s13662-017-1128-z.

[17] Y. W. Lee and G. H. Kim, "Hyperstability and stability of a logarithmic-type functional equation." Math. Stat., vol. 7, no. 1, pp. 25-32, 2019, doi: 10.13189/ms.2019.070104. 
[18] G. Maksa and Z. Pales, "Hyperstability of a class of linear functional equations." Acta Mathematica, vol. 17, no. 2, pp. 107-112, 2001.

[19] M. Piszczek, "Remark on hyperstability of the general linear equation." Aequ. Math., vol. 88, pp. 163-168, 2014, doi: 10.1007/s00010-013-0214-x.

[20] J. M. Rassias, "On approximately of approximately linear mappings by linear mappings." J. Funct. Anal., USA., vol. 46, pp. 126-130, 1982, doi: 10.1016/0022-1236(82)90048-9.

[21] T. M. Rassias, "On the stability of the linear mapping in Banach spaces." Proc. Amer. Math. Soc., vol. 72, pp. 297-300, 1978, doi: 10.1090/S0002-9939-1978-0507327-1.

[22] K. Ravi, J. M. Rassias, and B. V. Senthil Kumar, "Ulam stability of reciprocal difference and adjoint functional equations.” Aust. J. Math. Anal. Appl., vol. 8, no. 1, pp. 1-18, 2011.

[23] K. Ravi and B. V. Senthil Kumar, "Ulam-Gavruta-Rassias stability of Rassias reciprocal functional equation." Global J. Appl. Math. Math. Sci., vol. 3, no. 1-2, pp. 57-79, 2010.

[24] K. Ravi, E. Thandapani, and B. V. Senthil Kumar, "Stability of reciprocal type functional equations." PanAmerican Math. J., vol. 21, no. 1, pp. 59-70, 2011.

[25] S. Saejung and J. Senasukh, "On stability and hyperstability of additive equations on a commutative semigroup." Acta Math. Hungarica, vol. 159, pp. 358-373, 2019, doi: 10.1007/s10474-01900998-3.

[26] B. V. Senthil Kumar, Ashish Kumar, and P. Narasimman, "Ulam stability of radical functional equation in the sense of Hyers, Rassias and Gavruta." Int. J. Stat. Appl. Math., vol. 1, no. 4, pp. 6-12, 2016.

[27] B. V. Senthil Kumar and H. Dutta, "Non-Archimedean stability of a generalized reciprocalquadratic functional equation in several variables by direct and fixed point methods." Filomat, vol. 32, no. 9, pp. 3199-3209, 2018, doi: 10.2298/FIL1809199.

[28] B. V. Senthil Kumar and H. Dutta, "Approximation of multiplicative inverse undecic and duodecic functional equations." Math. Meth. Appl. Sci., vol. 42, pp. 1073-1081, 2019, doi: 10.1002/mma.5413.

[29] B. V. Senthil Kumar and H. Dutta, "Fuzzy stability of a rational functional equation and its relevance to system design." Int. J. General Syst., vol. 48, no. 2, pp. 157-169, 2019, doi: 10.1080/03081079.2018.1523904.

[30] B. V. Senthil Kumar and H. Dutta, "Fundamental stabilities of various forms of complex valued functional equations." in: Dutta H., Peters J. (eds) Applied Mathematical Analysis: Theory, Methods, and Applications. Studies in Systems, Decision and Control, Springer, Cham, vol. 177, pp. 29-59, 2020.

[31] B. V. Senthil Kumar, H. Dutta, and S. Sabarinathan, "Approximation of a system of rational functional equations of three variables." Int. J. Appl. Comput. Math., vol. 5, no. 3, pp. 1-16, 2019, doi: 10.1007/s40819-019-0626-y.

[32] S. M. Ulam, Problems in Modern Mathematics, Chapter VI. New York: Wiley-Interscience, 1964.

Authors' addresses

B. V. Senthil Kumar

Department of Information Technology, University of Technology and Applied Sciences, Nizwa, 611, Oman

E-mail address: senthilkumarenct.edu.om 
Khalifa Al-Shaqsi

Department of Information Technology, University of Technology and Applied Sciences, Nizwa, 611, Oman

E-mail address: khalifa.alshaqsienct.edu.om

\section{Hemen Dutta}

Department of Mathematics, Gauhati University, Guwahati, 781014 Assam, India

E-mail address: hemen_dutta08@rediffmail.com 